\title{
Valores, modos y modas en el ejercicio de la medicina
}

Publicado en "Educación médica Internacional", Vol. 9, Suplemento 1, XII-2006, pp. 15-20.

A la hora de considerar los problemas que afectan al ejercicio de la profesión médica no basta con considerarlos desde el punto de vista de lo que podríamos considerar la medicina misma sino que hay que tener también muy en cuenta una serie de propiedades del entorno cultural y social, porque la medicina, además de un saber y de un arte es, muy fundamentalmente, una función social básica. Dado que la sociedad occidental ha cambiado de manera muy radical en las últimas décadas sería realmente sorprendente que no hubiese desajustes y problemas en el ejercicio de una función tan llena de historia como la medicina clínica que, por otra parte, también ha sufrido sus mutaciones autónomas.

Los sociólogos han llamado la atención sobre el hecho de que en la sociedad contemporánea tiende a desdibujarse con gran rapidez casi cualquier vínculo inmediato entre palabras y cosas, incluso entre palabras y palabras. Estamos, de hecho, ante una sociedad muy plural y compleja en la que un cierto relativismo básico se ha convertido en una especie paradójica de valor indiscutible. A veces se llama reflexividad a este fenómeno, una supuesta libertad de pensamiento basada en una sospecha general que afecta a cualquier pretensión de verdad, que intensifica la confusión y que, en definitiva, hace bien real el temor de Juan de Mairena: en la mayoría de los casos ya no existe la verdad sino solo lo que dice Agamenón (cualquiera de los muchos Agamenones) o lo que dice el porquero (uno cualquiera de los infinitos porqueros). Si a esa enorme debilidad que afecta a buena parte de los conocimientos antes tenidos por sólidos se añade la fuerte politización que suele impedir en numerosísimos terrenos el debate sereno (porque se sabe desde el principio quién es el amigo y quién el enemigo), nos encontramos con que en el mundo contemporáneo el viejo oficio del intelectual se ha vuelto una auténtica rareza. En cualquier caso, este ambiente intelectual aconseja el refugio en el espacialismo (el pequeño 
grupo con el que podemos entendernos) y da lugar a una atomización de las cuestiones que favorece de modo preocupante la falta de diálogo. Cada corporación, cada oficio, cada individuo tiende a guiarse por su librillo y a defenderse del que pretenda darle cualquier clase de lecciones.

En esta atmósfera intelectual se tenderá a ver como un desafuero que alguien que no sea médico opine sobre problemas que afectan a los médicos, como por ejemplo las cuestiones de carácter ético. Se trata, sin embargo de problemas para los que la ciencia médica no tiene respuestas especialmente válidas, en ocasiones, aunque no siempre, porque esas respuestas no existen en ninguna parte. Sin embargo la reflexión desde fuera sobre los problemas de la práctica médica constituye un desafío intelectual de gran magnitud. Valgan estas líneas de pórtico para algunos de los problemas que precisan un tratamiento más detenido y constante.

El ejercicio de la medicina se realiza a través de una actividad profesional cuyo estatuto público ha ido adquiriendo importantes matices diferenciales a lo largo de una historia milenaria. Por mucho que miremos hacia el pasado o hacia culturas muy distintas a la nuestra será difícil encontrar sociedades, por primitivas que nos puedan parecer, en las que no existan personas que ejercen alguna función médica, que se preocupen de curar y de ayudar a quienes sufren. Muchas son las cosas que han cambiado muy profundamente, sin embargo, en el entorno que define la práctica médica, en las expectativas que suscita y en las demandas que recibe.

Pese a que los especialistas en historia tiendan a subrayar los cortes y las revoluciones como hitos decisivos en el desarrollo del conocimiento y en los cambios sociales, no es fácil deshacerse de la idea de que una actividad como la médica, que se ha ejercido de manera ubicua y con una continuidad apenas interrumpida en las más distinta épocas, no se ha desprendido nunca del todo de sus rasgos más sobresalientes en cada momento. Así podemos encontrar en el médico de hoy formas de comportamiento que vinculan el ejercicio de su profesión con etapas muy antiguas de la práctica médica, del mismo modo que son patentes, por el contrario, determinados patrones de comportamiento profesional que derivan de factores mucho más modernos, como, por ejemplo, el régimen jurídico en que se enmarca el ejercicio de la 
profesión o el componente tecnológico que ha ido adquiriendo buena parte de la práctica médica en nuestros días.

Esta convivencia de lo nuevo y de lo antiguo es inevitable, y es muy característica de los asuntos en los que se pone en juego los valores esenciales que dan sentido a la vida, unos valores que, incluso en el seno de nuestra cultura, no configuran una lista cerrada y de lectura inequívoca. En suma, la práctica médica está sometida, como cualquier otra institución social, a los vaivenes del tiempo, a las ataduras de la época. La mentalidad científica nos invita a ver en cada cambio un progreso y nos hace más difícil la comprensión del carácter no lineal de algunos cambios sociales que a veces, lejos de ser mejoras inequívocas suponen ciertos retrocesos o tienden a exhibir un cierto carácter cíclico, como ocurre notoriamente con las modas que van y vienen.

Apenas es posible pensar una actividad humana que esté completamente al abrigo de esa clase de oscilaciones, cuyo transcurso en el tiempo sea perfectamente lineal e inequívocamente progresivo, sin que existan otros factores de cambio que los puramente lógicos o racionales. Un análisis irónico de cómo se produce la renovación en la ciencia, que se atribuye habitualmente a Max Planck, afirma que las verdades científicas no triunfan convenciendo a sus oponentes y haciéndoles ver la luz, sino, más bien, porque los oponentes se mueren y una nueva generación crece familiarizándose con la proclamación y la aceptación de las verdades nuevas.

Cuando cualquier problema dista de estar enteramente claro, el problema mismo se convierte en una fuente de disputas inacabables. Sin embargo, al menos en ocasiones, cuando esas disputan se acaban es peor, porque puede ocurrir que emerja un consenso a la Planck sin demasiados argumentos decisivos en su favor. La razón es sencilla: la gente necesita creer y quiere creer que las cosas son como le parece que son y es muy difícil mantener en alto la guardia para distinguir con nitidez lo que se sabe de lo que se cree saber. Nadie comprueba, nadie podría hacerlo, todas las cosas que se le enseñan como verdaderas, de modo que basta un tiempo no muy largo para que un error de apreciación indebidamente generalizado pueda convertirse en dogma. La ciencia sólo progresa adecuadamente cuando se hace prácticamente inmune a esa pasión por creer, a ese deseo de confiar en que ya se sabe todo. 
El médico dedicado a la clínica (no, por supuesto, en el del médico dedicado a la investigación), sea cual sea la calidad de su preparación científica, no es propiamente un científico, no tiene que dirigir sus pasos al establecimiento de una verdad abstracta que puede resultar inútil para su paciente sino a la curación de la persona concreta que tiene a su cargo. En el trabajo del médico el carácter práctico tiene que predominar necesariamente sobre su carácter teórico. Se halla, por tanto, frente a la necesidad de formular juicios prácticos, aquellos sobre cuya dificultad especial ya llamó la atención precisamente uno de los investigadores que más contribuyó a hacer de la medicina se convirtiera en una Ciencia. Ésta es la reflexión de Claude Bernard:

"En medicina, es posible también elevarse a las generalidades más abstractas, ya colocándose en el punto de vista del naturalista y considerando a las enfermedades como especies morbosas que trata de definir y clasificar nosológicamente, ya porque, partiendo del punto de vista fisiológico, se considere que la enfermedad no existe, en el sentido de que no sería más que un caso particular del estado fisiológico. No hay dudad de que todas estas opiniones son rayos de luz que nos dirigen $\mathrm{y}$ nos son útiles. Pero si nos entregamos exclusivamente a esta contemplación hipotética, bien pronto volveríamos la espalda a la realidad; y eso sería, en mi opinión, interpretar mal la verdadera filosofía científica, al establecer una especie de oposición o exclusión entre la práctica que exige el conocimiento de las particularidades y las generalizaciones precedentes que tienden a integrar todo en el todo. En efecto, el médico no es, modo alguno, el médico de los seres vivos en general, ni siquiera el médico del género humano, sino el médico del individuo humano, $\mathrm{y}$ además el médico de un individuo en determinadas condiciones morbosas peculiares a él, y que constituye lo que se ha llamado su idiosincrasia"1. (Cursivas del autor.)

En ese contexto, el médico está necesariamente abocado a dar por buenas cuantas cosas le han sido enseñadas y a hacerlo con la única

\footnotetext{
${ }^{1}$ Bernard Cl. Introducción al estudio de la medicina experimental (trad. al español de Luis Alberti). En: Laín Entralgo P (ed.). Claudio Bernard. Madrid: Ediciones el Centauro; Clásicos de la Medicina. 1947, p. 258. (Publicada originalmente en 1865.).
} 
libertad que le pueden permitir los siempre estrechos límites de su experiencia personal. El médico está especialmente inclinado a creer en la verdad de unos remedios cuya validez no siempre ha podido comprobar de primera mano (y eso dejando de lado las paradojas que acompañan a cualquier análisis de los procesos de verificación). El médico es, antes que un investigador o un teórico cuyo buen oficio consiste en la capacidad de poner en duda buena parte de lo que se le ha enseñado, un miembro de una profesión que ha de actuar necesariamente conforme a unos saberes fundados con mayor o menor rigor, a unas normas establecidas con mayor o menor acierto y a una experiencia clínica personal más o menos rica. Incluso Pío Baroja (1872-1956), del que por cierto este año celebramos el cincuenta aniversario de su fallecimiento, que ejerció escasamente un año, pudo darse cuenta, en tan corto período, de que el ejercicio de la medicina es algo más complejo que la rigurosa adquisición de unos conocimientos teóricos. La falta de tacto humano o de habilidad para entender las circunstancias de todo tipo que rodean al padecimiento físico puede crear verdaderos problemas a un paciente concreto: "A veces molestaba uno a los pacientes sin quererlo y sin pensarlo. Muchas viejas enfermas, aunque no se hallaban graves, le decían a uno que querían confesarse y comulgar. Si entonces se les decía que no se encontraban en estado tan grave, resultaba que se incomodaban. Al parecer, diciendo que se encontraban mal eran más atendidas y cuidadas”.

El médico está vinculado por las ideas vigentes acerca de la salud, un concepto de difícil precisión como no sea mediante una definición casi vacía de puro obvia y exagerada. Y debe hacerlo con los medios que le proporciona el entrenamiento en una profesión especialmente ligada a la tradición, que sabe tanto o más por vieja que por diabla. La experiencia histórica da una cierta seguridad, pero no lo es todo. Muchas de las exigencias contemporáneas en materia de salud apenas tienen antecedentes. Baste con considerar la complicación que introduce en el panorama de la práctica clásica de la medicina la paradoja de que el incremento global de la duración media de la vida (dato que, sean cuales sean las precisas causas históricas, significa un éxito de las políticas de higiene y sanidad) trae consigo un notable aumento de las necesidades sanitarias de una población de edad media muy avanzada.

\footnotetext{
2 Baroja P. Familia infancia y juventud. Madrid: Caro Raggio, editor. 1982, pp. 366-367.
} 
El desarrollo científico, el desarrollo de la higiene y el desarrollo económico han diversificado y dificultado enormemente las exigencias sociales que se plantean a los médicos y han traído consigo una notable complicación del entorno ético y profesional en el que se desenvuelve la medicina: mayores exigencias, mayores controles, mayor conflictividad y, como consecuencia, en muchas ocasiones, mayor frustración.

El médico ya no puede ser un chamán al que nadie discute, aunque haya de conservar algo de esa vieja sabiduría capaz de infundir respeto y dotar de credibilidad a su trabajo como condición previa de eficacia. $\mathrm{Su}$ autoridad se ve reforzada por el prestigio de la ciencia y el predicamento de la tecnología que utiliza, pero se ve también debilitada cuando, como ocurre en la cultura posmoderna, el prestigio de la ciencia comienza a ponerse en solfa o se repara en el carácter inhumano de muchas tecnologías médicas. Como si fuese consciente de ese déficit de legitimidad (el chamán ya no tiene público y la ciencia tampoco es lo que era), la sociedad tiende a reforzar la autoridad del médico confiriéndole un status de autoridad civil, haciendo de él un funcionario de la salud.

No es fácil para nadie manejar unas fuentes de legitimidad tan inconexas y, en ocasiones, algo más que levemente contradictorias. El paciente debe seguir los consejos del médico porque se fía personalmente de él (de su chamán), pero tiende a seguirlos por su saber o por su autoridad puesto que, al fin y al cabo, el médico es quien decide si tiene que ir a trabajar o puede quedarse en casa o quien determina otra serie de asuntos que mucho le importan, como, por ejemplo, la cuantía de una indemnización o su capacidad para asumir ciertas funciones.

La generalización de los servicios sanitarios ha convertido al médico, por otra parte, en el centro de un sistema de gasto de dimensiones colosales en el que todo parece estar un poco fuera de control. El aumento del personal destinado a los servicios de sanidad parece ser una constante de los últimos años, y ello independientemente del carácter público (Europa) o privado (EEUU) de los sistemas de salud. Ello ha provocado una suerte de reprivatización de la práctica médica puesto que, al fin y a la postre, el paciente se encuentra a solas con su médico pero, además, porque los gestores de los sistemas sanitarios 
han comenzado a forzar a los médicos a tratar a sus usuarios como si fueran consumidores o clientes de un servicio cualquiera.

Si a ello se añade el efecto superficial provocado por las filosofías antiautoritarias (más terminológico que real, en cualquier caso) que se han traducido en un rechazo, al menos retórico, del llamado paternalismo médico, y el auge de las preocupaciones bioéticas centradas en la doctrina de la autonomía de los pacientes, se hace fácil comprender que el médico se haya visto convertido con alguna frecuencia en una especie de asesor, en alguien a quien el cliente pregunta para hacer luego lo que mejor le parezca. Todo ello configura un panorama en el que, como ha escrito José Luis Puerta3, la medicina se ha convertido, de alguna manera, en un bien de consumo.

Si hago la enumeración sumaria de algunos de los factores sociológicos y políticos que condicionan el complejísimo entorno de la práctica médica contemporánea es para advertir, a continuación, que todo este panorama requeriría una reflexión de conjunto muy a fondo y, muy probablemente una redefinición de los principios básicos que han inspirado la práctica médica tradicional, digamos, hasta la Segunda Guerra Mundial.

Es imposible que nadie sea a la vez un buen chamán, un científico puntero, una autoridad civil prestigiosa y un asesor profesional competente. Cualquier persona a quien se cargue con la mitad de esas responsabilidades cumplirá chapuceramente con, al menos, una de ellas. Urge, por tanto, un debate de fondo sobre las funciones de la medicina en la sociedad del siglo XXI y no servirá de nada hacer como si no pasase nada y estuviésemos en 1930 con una sociedad que respeta y admira a los médicos y con unos profesionales que pueden cumplir, con sacrificio, desde luego, pero sosegadamente, con su función.

Las páginas de los periódicos se llenan a diario con informaciones sanitarias, con reclamaciones, con denuncias. Muchos pacientes consultan Internet previamente a acudir al médico. Nada es lo que era y tenemos que preguntarnos qué queremos que sea. No sirve de nada conformarse con ilusiones. Si no se abordan a fondo las cuestiones pendientes y se acometen las reformas necesarias la medicina seguirá conservando gran parte de su merecido prestigio, pero crecerá la

\footnotetext{
${ }^{3}$ Puerta JL. Editorial: El verdadero debate. Revista de Humanidades. 2005;4(2):181-184.
} 
conflictividad, aumentarán los costes y se perderán oportunidades de hacer las cosas mejor.

Seguir pensando que la acción del médico se justifica en función de la honorabilidad o el altruismo de sus fines y en la existencia de algo así como una conciencia médica objetiva que se obtiene de modo casi insensible y automático con una buena preparación profesional, es renunciar a entender la complejidad del mundo en que vivimos y vamos a vivir. Está bastante claro que, al menos desde hace medio siglo (trasplantes, diálisis, avances bioquímicos y en biología molecular, etc.), el campo de la medicina no cesa de plantear problemas de índole moral que no pueden ignorarse o subjetivarse4.

Preguntarnos qué podemos esperar de la medicina y qué deberíamos hacer para conseguirlo supone replantear a fondo cada una de las dimensiones del conjunto del sistema sanitario para tratar de detectar y corregir sus incoherencias sus disfunciones y sus efectos perversos, que de todo abunda. No es una tarea que nadie pueda acometer en solitario ni tampoco es algo que, pese a su indudable repercusión política, quepa dejar en las solas manos de los políticos que, la mayoría de las veces se dejan llevar por la mera rutina y por la apariencia sin atreverse a plantear cuestiones que puedan suscitar el rechazo de los electores.

En el conjunto de cuestiones que ha de suscitar este análisis brilla con especial luz propia la necesidad de establecer un marco legal muy preciso para las decisiones médicas que tienen fuertes implicaciones éticas. Los médicos no deberían dejarse llevar por la tentación de seguir haciendo lo que les parece bien sin que los afectados puedan opinar, dejándose llevar por la pretensión un poco quimérica de que existe una suerte de ética médica que solo ellos conocen y que a nadie es lícito poner en cuestión. Esta es una actitud que tal vez pudiera haberse defendido en otros momentos en que la serie de los principios éticos gozaba de un mayor consenso que el que ahora es llevadero pero que ahora es totalmente inane. La capacidad de decisión del médico no puede ignorar lo que establece la ley ni lo que mandan los correspondientes protocolos profesionales de actuación. Tanto la ley como esos protocolos gozan y deben gozar de un estatuto público cuya

\footnotetext{
${ }^{4}$ González Quirós, J. L.: Incertidumbre y riesgo en biomedicina. Argumentos de razón técnica. 2002; 5:177-206.
} 
existencia cumple dos funciones esenciales: en primer lugar, obligar a que los profesionales certifiquen con precisión determinadas decisiones y actos médicos y, en segundo término, que puedan usarse para legitimar reclamaciones fundadas.

La autoridad del médico no puede consistir en un poder capaz de evitar cualquier examen de sus actos. El médico, que se las ve con la vida y la muerte de sus pacientes, tiene que estar socialmente protegido frente a reclamaciones quiméricas para poder obrar con la libertad de juicio que requiere cualquier profesión intelectual, pero ello no puede entenderse en el sentido de que su actuación quede libre del sometimiento a ciertas normas establecidas por prudencia y por la experiencia profesional acumulada. No está de más recordar, aunque la anécdota pueda rozar la caricatura, que allá por 1870, cuando Pasteur alertó al personal sanitario de que podían estar transportando en sus manos un microbio desde las mujeres enfermas a las sanas, un médico se sintió ultrajado por poner en duda el honor de la profesión y lo retó a un duelo. Si bien la reacción no tuvo esta teatralidad, la respuesta que el gran cirujano y ginecólogo estadounidense, Charles Delucena Meigs (1792-1869), al que se le debe una de las técnicas de histerectomía más ampliamente usadas, dio al profesor de Harvard Oliver Wendell Holmes 5 (18091894), cuando éste hizo notar la alta tasa de fiebre puerperal que se registraba en la práctica de muchos médicos, estaba inspirada en esa misma mentalidad que cree poder hallar en la honorabilidad personal (hoy diríamos en la conciencia profesional de cada cual) una garantía por encima de cualquier escrutinio: "He practicado la obstetricia desde hace muchos años, he atendido varios miles de parturientas y he asistido a repetidas epidemias de fiebre puerperal, tanto en ciudades como en hospitales. Después de toda esta experiencia, sin embargo, no encuentro - tras una rigurosa reflexión y autoexamen- el más mínimo motivo para suponer que yo haya en alguna ocasión transportado la enfermedad de un sitio a otro en ningún caso... A lo largo de mi vida profesional he practicado muchas necropsias con el objeto de investigar la fiebre puerperal, pero nunca he suspendido mi actividad como tocólogo por este motivo. En todo caso, estoy seguro de que yo no he sido el medio de su transmisión... las manos de un caballero están

\footnotetext{
5 Sobre este interesante personaje, véase: Prieto S. Oliver Wendell Holmes (1809-1894). Estetoscopio y Letras. Revista de Humanidades. 2006;5(1):133-140.
} 
limpias"6. (Las cursivas son mías.)

Esas normas a las que me vengo refiriendo no limitan la libertad del médico precisamente porque deben fijar muy estrictamente el ámbito en el que es el único responsable y sus decisiones están fuera de discusión. De ninguna manera, esta situación es incompatible, todo lo contrario, con el derecho que el paciente tiene a ser el protagonista de su enfermedad y, por tanto, a participar en las decisiones que conciernen a su salud. Y éste fue precisamente el gran paso que se dio en 1973, cuando la American Hospital Association publicó la primera "Carta de derechos del paciente" (Patient's Bill of Rights), que favoreció que el equilibrio de autoridad se desplazara de los médicos a los pacientes y se empezasen a desterrar los modos paternalistas en las consultas. No se olvide, sin embargo, que es más fácil desterrar un defecto en la teoría que no cometerlo en la práctica, de modo que lo que ocurre en muchas ocasiones es que la autonomía del paciente es más retórica que efectiva.

En otros momentos de la historia ha sido posible, sin duda, un ejercicio profesional más independiente de controles, más absolutista, por decirlo de algún modo, pero hoy en día se nos antoja inconcebible, tanto por la complejidad de la profesión médica como por la actitud que nuestra sociedad tiene hacia la sanidad. Es el conjunto de factores que determinan la práctica médica el que ha cambiado y está cambiando con mucha celeridad. Es imposible pensar en un ejercicio ético de la profesión médica que no tome en cuenta la gravedad y novedad de esta clase de cambios, aunque ello suponga que, en muy contadas ocasiones, el médico haya de trabajar sometido a un escrutinio casi constante. Entre los compromisos que el médico adquiere al ponerse al servicio de los demás ni figura, ni puede figurar de ninguna manera el privilegio de no dar explicaciones.

\section{José Luis González Quirós \\ Instituto de Filosofía, CSIC \\ jlgonzalezquiros@gmail.com \\ http://jlgonzalezquiros.es/}

\footnotetext{
${ }^{6}$ Meigs CD. On the Nature, Sings, and Treatment of Childbed Fevers. Philadelphia. 1859, p. 102 y 104. La cita ha sido tomada de: Sharpe VA y Faden AI. Medical Harm. Historical, conceptual and ethical dimensions of iatrogenic illnes. Cambridge: Cambridge University Press. 1998, p. 154.
} 